\title{
RELACIÓN ENTRE COMPORTAMIENTOS DE HIPER-REACTIVIDAD SENSORIAL Y ALTERACIONES DEL SUEÑO EN LA INFANCIA
}

\section{RELATIONSHIP BETWEEN SENSORY HYPER-REACTIVITY BEHAVIOURS AND SLEEP DISTURBANCES IN CHILDHOOD}

\author{
Alberto León-Collado. ${ }^{1}$ Stella Martín-Rubiño. ${ }^{2}$ Rafael Ruiz-Cañete. ${ }^{3}$ Cristina De Abajo- \\ Pinteño. ${ }^{4}$ Saray Parrón-Collar. ${ }^{5}$ Rubén Barroso-Canales. ${ }^{6}$
}

\begin{abstract}
RESUMEN
Descanso y Sueño son considerados por la Asociación Americana de Terapia Ocupacional (AOTA, 2017) como una de las áreas de la ocupación humana, definiéndola como aquellas actividades relacionadas con la obtención de descanso reparador y sueño para apoyar un compromiso saludable y activo en otras ocupaciones. La literatura relacionada vincula la calidad y duración del sueño con el desarrollo de habilidades cognitivas como la consolidación de la memoria emocional y el aprendizaje. El objetivo de este estudio es evidenciar cómo la hiperreactividad sensorial puede relacionarse con la aparición de alteraciones del sueño en la infancia. Con las puntuaciones obtenidas en los cuestionarios Perfil Sensorial-2 Breve de Dunn (Dunn, 2016) y la Escala de Trastornos del Sueño de Bruni (Bruni, et al., 2O13) se elaboraron análisis correlacionales de Pearson. Los padres y/o madres de 92 menores de entre 3 y 14 años de edad con alteraciones en el neurodesarrollo y otros diagnósticos infantiles completaron estos cuestionarios durante el primer semestre de 2019. Se encontraron puntuaciones de correlación altamente significativas entre las alteraciones en el sueño y los patrones conductuales del procesamiento sensorial de tipo: Búsqueda $(p<0,01)$, Evitación $(p<0,01)$, Sensibilidad $(p<0,01)$ y Sensorial $(p<0,01)$.
\end{abstract}

1 Terapeuta Ocupacional Universidad Complutense de Madrid. Máster en Neurociencias y Biología del Comportamiento Universidad Pablo de Olavide. Certificación en Integración Sensorial University of Southern California. Institución. Servicio de Terapia Ocupacional Infantil S.T.O.I. Jerez C.E. Las Bodegas, Calle Añada, nº16. Jerez de la Frontera, Cádiz. España. info@stoi.es. +34 665212357

2 Terapeuta Ocupacional Universidad de Granada. Certificación en Integración Sensorial University of Southern California. Institución. Servicio de Terapia Ocupacional Infantil S.T.O.I. Jerez C.E. Las Bodegas, Calle Añada, nํ⒗ Jerez de la Frontera, Cádiz. España. stellato@stoi.es. +34 687232503

3 Terapeuta Ocupacional Universidad de Zaragoza. Certificación en Integración Sensorial University of Southern California. Institución. Servicio de Terapia Ocupacional Infantil S.T.O.I. Jerez Avenida de Chipiona, C.E. Las Bodegas, Calle Añada, nº16. Jerez de la Frontera, Cádiz. España. rafaelto@stoi.es +34687254250

4 Terapeuta Ocupacional Universidad de Burgos. Certificación en Integración Sensorial University of Southern California. Institución. Servicio de Terapia Ocupacional Infantil S.T.O.I. Jerez C.E. Las Bodegas, Calle Añada, nº16. Jerez de la Frontera, Cádiz. España. cristinato@stoi.es $+34680503333$

5 Terapeuta Ocupacional Universidad de Castilla La Mancha. Certificación en Integración Sensorial University of Southern California. Institución. Servicio de Terapia Ocupacional Infantil S.T.O.I. Jerez Avenida de Chipiona, C.E. Las Bodegas, Calle Añada, nº16. Jerez de la Frontera, Cádiz. España. sarayto@stoi.es +34 626913242

6 Terapeuta Ocupacional y Máster oficial de Trastornos de la Comunicación por la Universidad de Salamanca. Certificación en Integración Sensorial University of Southern California. Institución. Fundesalud (Fundación para la Formación e Investigación de los Profesionales de la Salud de Extremadura). Avenida de Extremadura, 22. Navas del Madroño, Cáceres. España. rubenbc.to@gmail.com +34 646820366 
La presencia de comportamientos de hiperreactividad sensorial se vincula a la presencia de alteraciones del sueño. La evaluación y el tratamiento en esta área de ocupación cobran vital importancia en la intervención desde Terapia Ocupacional.

\title{
PALABRAS CLAVE
}

Terapia Ocupacional, desarrollo infantil, sueño.

\begin{abstract}
Rest and Sleep are considered by The American Occupational Therapy Association (AOTA, 2017) as one of the occupational areas. It is defined as activities related to obtaining restorative rest and sleep to support healthy and active engagement in other occupations. Related literature links the quality and duration of sleep with the development of cognitive skills such as the consolidation of emotional memory and learning. The objective of this study is to support how sensory hyperresponsiveness can be related to the appearance of sleep disturbances in childhood. The scores obtained in Dunn's Short Sensory Profile-2 questionnaire (Dunn, 2016) and the Bruni Sleep Disorders Scale (Bruni, et al., 2013), were correlated using Pearson's analysis. The fathers and / or mothers of 92 minors between 3 and 14 years of age with neurodevelopmental disorders and other childhood diagnoses completed these questionnaires during the first semester of 2019. Highly significant correlation scores were found between sleep disorders and the behavioural patterns of sensory processing of type: Seeking ( $p<0.01)$, Avoiding $(p<0.01)$, Sensitivity $(p<0.01)$ and Sensory $(p<0.01)$. The presence of sensory hyper-responsive behaviours suggests it is linked to the presence of sleep disturbances. The evaluation and the treatment in this area of occupation are vitally important in the intervention from Occupational Therapy.
\end{abstract}

\section{KEYWORDS}

Occupational Therapy, child development, sleep.

Recepcionado: 09/09/2020

Aceptado: 26/11/2020

\section{INTRODUCCIÓN}

Aquellas actividades relacionadas con la obtención de un descanso reparador y sueño para apoyar una participación activa y saludable en otras ocupaciones, es la definición usada por la Asociación Americana de Terapia Ocupacional (АОтA, 2017) para describir el área ocupacional de Descanso y Sueño, siendo la única área ocupacional, que ya en su definición relaciona a dichas actividades con un mejor desempeño en otras áreas de ocupación.

Además, tradicionalmente desde la Ciencia de la Ocupación y la Terapia Ocupacional se ha descrito y analizado el complejo rol del sueño y el impacto que tiene en la ocupación humana y el bienestar, apareciendo reflejado tanto en los primeros marcos de trabajo (Meyer, 1922; Slagle, 1922) como en modelos más contemporáneos de la disciplina como el Modelo de Ocupación Humana (Kielhofner, 2008).
Sin embargo, son escasas las revisiones sistemáticas y otras publicaciones con alto nivel de evidencia realizadas desde la Terapia Ocupacional en la intervención de las dificultades en la participación en actividades de descanso y sueño, hecho que pudiera sugerir el escaso abordaje que, en la actualidad, se está haciendo sobre esta área de ocupación en las intervenciones de Terapia Ocupacional. En los últimos años han sido dos las revisiones sistemáticas que han recogido las publicaciones realizadas sobre intervenciones en Terapia Ocupacional y alteraciones del sueño, de las cuales sólo una recogía estudios realizados en población infantil (Eris y Andrew, 2018). El sueño es considerado además de un área de ocupación dentro de nuestra disciplina, como una condición neurofisiológica, definiéndose como el estado fisiológico con abolición periódica de la conciencia vigil y la reducción de las respuestas a los estímulos ambientales. Es un fenómeno rítmico y reversible que se acompaña de cambios en diversas funciones ejecutivas (Idiazábal-Aletxa y Aliagas-Martínez, 2009). No 
es un proceso pasivo, es un estado activo y dinámico complejo que tiene gran impacto sobre la salud, el funcionamiento durante la vigilia y el desarrollo. Así pues, es un proceso biológico que incorpora componentes conductuales y sociales (Cruz, 2018).

Existe una escasa consolidación en la evidencia en relación a las dificultades o alteraciones en el sueño y su impacto en el desarrollo infantil, (Paavonen, et al., 2010) aunque son numerosos los estudios y publicaciones que muestran relación entre las alteraciones en el sueño y la consolidación del aprendizaje en la infancia (Cousins, Rijn, Ong, Wong y Chee, 2019; Gruber, Somerville, Enros, Paquin, Kestler y Gillies-Poitras, 2014; Reale, Guarnera y Mazzone, 2014; Geiger, Achermann y Jenni, 2010).

Se encuentra mayor evidencia en la literatura cuando se habla de la relación entre alteraciones del sueño de larga duración y la consolidación de la memoria emocional así como un aumento en la aparición de conductas de irritabilidad e impulsividad en niños. Esto es debido a la continua actividad del Locus Coeruleus durante la fase de Movimientos Oculares Rápidos (MOR) también conocido por sus siglas en inglés, fase REM (Rapid Eye Movement), que impide una adaptación de la amígdala durante esta fase del sueño (Wassing, Lakbila-Kamal, Ramautar, Stoffers, Schalkwijk y Van Someren, 2019), estando así ambas estructuras del Sistema Nervioso Central íntimamente vinculadas a las bases neurofisiológicas de la hiperreactividad sensorial (Miller, Anzalone, Lane, Cermak y Osten, 2007).

La hiperreactividad sensorial se define como la respuesta a experiencias sensoriales de forma más rápida, más intensa o con una mayor duración que las personas que tienen una respuesta sensorial típica (Miller, et al., 2007).

Además, las respuestas observadas asociadas a la hiperreactividad sensorial pueden ser conductas que van desde una mayor actividad y negatividad ante el estímulo, como la impulsividad o la agresividad, o más pasivas como la abstención o la evitación de la sensación, existiendo una implicación directa del Sistema Nervioso Simpático (sNS). Las conductas típicamente asociadas a una respuesta excesiva del SNS debido a una hiperreactividad sensorial son: lucha, evitación, susto o bloqueo (Miller, et al., 2007; Miller, et al., 1999).
Diferentes estudios sugieren una significativa relación entre problemas de hiperreactividad sensorial y problemas del sueño en niños en edad escolar de desarrollo típico (Foitzik y Brown, 2018; Shochat, Tzischinsky y Engel-Yeger, 2009; Vasak, Williamson, Garden y Zwicker, 2015) y en niños con un diagnóstico de dermatitis atópica (Shani-Adir, Rozenman, Kessel y Engel-Yeger, 2009).

El objetivo principal de este estudio es evidenciar que la hiperreactividad sensorial puede relacionarse con la aparición de alteraciones del sueño en la infancia. Además, pretende apoyar la evidencia existente sobre la relación entre la aparición de comportamientos vinculados a la hiperreactividad sensorial en niños y el mantenimiento de problemas del sueño en una población infantil con alteraciones en el neurodesarrollo y otros diagnósticos (Abril, Méndez, Sans y Valdizán, 2001; Allik, Larsson y Smedje, 2006; Brown, 1996; Cramer, Waldorp, Van derMaas y Borsboom, 2010; Ghorbanpour, Hosseini, Akbarfahimi y Rahgozar, 2019; Goldman, Surdyka, Cuevas, Adkins, Wang y Malow, 2009; Idiazábal-Alexta y Aliagas-Martínez, 2009; Mazurek y Petroski, 2015; O'Brien, et al., 2003; Valdizán, 2005).

\section{MÉTOdo}

Este es un estudio transversal que busca correlacionar los resultados obtenidos mediante el cuestionario Perfil Sensorial-2 Breve (PS-2 Breve) (Dunn, 2016) y la versión traducida y adaptada al castellano de la Escala de Trastornos del Sueño para Niños de Bruni, conocida por sus siglas en inglés Sleep Disturbances Scale for Children (SDSC) (Bruni, et al., 2013; Ministerio de Sanidad, Política Social e Igualdad, 2013), para conocer si existen relaciones estadísticamente significativas entre las puntuaciones obtenidas de ambos cuestionarios. Se escogió la opción de realizar este tipo de estudio debido a la disponibilidad de acceso a la muestra.

Ambos cuestionarios fueron contestados por padres y/o madres de una muestra de 92 niños y niñas con edades comprendidas entre los 3 y los 14 años de edad que presentan diferentes diagnósticos médicos y que acuden a un programa de intervención en Terapia Ocupacional en las instalaciones del Servicio de Terapia Ocupacional Infantil S.T.O.I. Jerez de la Frontera (Cádiz, España) donde desempeña su labor profesional el grupo de investigación autor de este artículo. Quedaron 
excluidos del estudio aquellos niños y niñas que mantienen un tratamiento psicofarmacológico (hipnóticos, psicoestimulantes, benzodiacepinas y antipsicóticos) o que toman algún tipo de fármaco anticonvulsivo dos horas antes de ir a la cama. Los cuestionarios fueron completados en una sola ocasión durante el primer semestre del año 2019 de forma presencial y bajo la supervisión de los investigadores.

Se entregó un documento de consentimiento informado a los padres y/o madres de los menores incluidos en la muestra en el que se detallaban todas las características del estudio anteriormente descritas. No se consideró necesario realizar un documento para informar del proceso de estudio a los menores incluidos en la investigación debido al tipo de herramientas usadas para la recopilación de información y a las características específicas de la muestra.

En la Tabla 1 se desglosa la muestra según el tipo de diagnóstico médico que presenta cada participante.

Con los resultados obtenidos en ambos cuestionarios se realizaron análisis correlacionales de Pearson.

TABLA 1 RELACIÓN DE DIAGNÓSTICOS INCLUIDOS EN EL ESTUDIO

\begin{tabular}{lll}
\hline Diagnóstico & $\begin{array}{l}N^{\mathbf{~}} \\
\text { participantes }\end{array}$ & Porcentaje \\
\hline Discapacidad Intelectual & 6 & $7 \%$ \\
Trastorno Espectro Autismo & 59 & $64 \%$ \\
Síndrome de Rett & 1 & $1 \%$ \\
Parálisis Cerebral infantil & 5 & $5 \%$ \\
Síndrome X-Frágil & 1 & $1 \%$ \\
Síndrome de Down & 5 & $5 \%$ \\
Esclerosis Tuberosa & 1 & $1 \%$ \\
Otros Síndromes Genéticos & 9 & $10 \%$ \\
Daño Cerebral & 1 & $1 \%$ \\
Trastorno por Déficit de & 2 & $2 \%$ \\
atención e hiperactividad & 2 & $2 \%$ \\
Retraso madurativo & 2 & $100 \%$ \\
TOTAL & 92 &
\end{tabular}

Número de participantes en el estudio según su diagnóstico y porcentaje de este en el estudio.
El cuestionario PS-2 Breve es una herramienta de evaluación, traducida, validada y estandarizada en población española diseñada para ayudar a evaluar los patrones de procesamiento sensorial del niño en contextos como el colegio, el hogar y la comunidad. La versión breve se compone de 34 ítem extraídos del Perfil Sensorial 2. Estos ítem son altamente discriminativos, dan una información rápida y son frecuentemente usados en programas de cribado e investigación. El PS-2 Breve proporciona seis puntuaciones: cuatro que se corresponden con los patrones sensoriales del modelo de procesamiento sensorial propuesto por Dunn (Búsqueda Sensorial, Evitación Sensorial, Sensibilidad Sensorial y Registro Sensorial) y dos puntuaciones de resumen, una de los ítem sensoriales y otra de los ítem conductuales (Dunn, 2016).

La SDSC (Ministerio de Sanidad, Política Social e Igualdad, 2013) es una herramienta de evaluación traducida, validada y estandarizada en población española recomendada para la detección de alteraciones del sueño en la infancia. Consta de 26 ítem valorados según una escala tipo Likert. Las puntuaciones clasifican las alteraciones del sueño en la infancia en 7 categorías: Problemas en el inicio y el mantenimiento del sueño; Síndrome de piernas inquietas/Síndrome movimiento periódico de extremidades/ Dolores de crecimiento; Trastornos respiratorios del sueño; Desórdenes de la alerta; Alteraciones del tránsito vigilia/sueño; Somnolencia diurna excesiva/déficit; Hiperhidrosis del sueño. Para el cómputo de datos de este estudio, sólo se usó la puntuación global del cuestionario. Dicho cuestionario está contemplado y aconsejado como herramienta para la detección de problemas y alteraciones del sueño en la infancia y la adolescencia en la Guía de Práctica Clínica sobre Trastornos del Sueño en la Infancia y la Adolescencia en Atención Primaria (Ministerio de Sanidad, Política Social e Igualdad, 2013), desarrollado por el Sistema Nacional de Salud del Gobierno de España.

\section{Resultados}

Una vez obtenidos los datos de ambos cuestionarios con las puntuaciones de la muestra, se realizaron análisis estadísticos tipo correlación de Pearson, mediante el uso del software IBM SPSS ${ }^{\circledast}$.

Para la realización de la correlación estadística de Pearson se incluyeron únicamente aquellos cuadrantes y 
TABLA 2 CORRELACIÓN DE PEARSON SDSC Y FACTORES DEL PS-2 BREVE (BÚSQUEDA, EVITACIÓN, SENSIBILIDAD) Y LA SECCIÓN SENSORIAL.

\begin{tabular}{llllll}
\hline Puntuaciones & Búsqueda & Evitación & Sensibilidad & Sensorial \\
\hline SDSC & Corr.de Pearson &, $414^{* *}$ &, $358^{* *}$ &, $389^{* *}$ &, $329^{* *}$ \\
& Sig. (bilateral) &, 000 &, 000 &, 000 &, 001 \\
& $N$ & 92 & 92 & 92 & 92 \\
\hline
\end{tabular}

**. La correlación es significativa al nivel 0,01 (bilateral).

secciones del PS-2 Breve que según la literatura revisada para este estudio se vinculan directamente con la hiperreactividad sensorial, estos son: Búsqueda Sensorial, Evitación Sensorial y Sensibilidad Sensorial, así como la sección Sensorial

Los resultados obtenidos se muestran en la Tabla 2 de este artículo, de la que se destaca una correlación bilateral altamente significativa entre puntuaciones altas obtenidas en la SDSC y el cuadrante de Búsqueda Sensorial $(p=0,000)$, SDSC y el cuadrante de Evitación Sensorial $(p=0,000)$, SDSC y el cuadrante Sensibilidad Sensorial $(p<0,001)$ y SDSC y la sección Sensorial $(p=$ $\mathrm{O}, \mathrm{OOO}$ ). Estos resultados dan validez a la hipótesis planteada en el estudio.

Sin embargo, los valores del índice $r$ establecen una correlación bilateral de escasa a moderada entre las puntuaciones de la SDSC y el cuadrante de Búsqueda Sensorial $(r=0,414)$, SDSC y el cuadrante de Evitación Sensorial $(r=0,358)$, SDSC y el cuadrante de Sensibilidad Sensorial $(r=0,389)$ y SDSC y la sección Sensorial $(r=0,329)$.

\section{Discusıón}

Los resultados obtenidos sugieren que altas puntuaciones en los cuadrantes de Búsqueda Sensorial, Evitación Sensorial, Sensibilidad Sensorial y en la sección Sensorial del PS-2 Breve, se vinculan con unas puntuaciones altas en el cuestionario SDSC, así como a la inversa: altas puntuaciones en el cuestionario SDSC se vinculan con puntuaciones altas en los cuadrantes y sección Sensorial del PS-2 Breve incluidos en este estudio. Tras la revisión de las publicaciones relacionadas, estos resultados se asemejan a otros estudios similares con muestra de población normotípica que usan otras escalas de medición diferentes a las de este estudio (Foitzik y Brown, 2018; Shani-Adir, Rozenman, Kessel y Engel-Yeger, 2009; Shochat, Tzischinsky y Engel-Yeger, 2009; Vasak, Williamson, Garden y Zwicker, 2015).

Esto sugiere que las limitaciones en la participación en actividades de sueño pueden estar relacionadas con las conductas asociadas a la hiperreactividad sensorial, así como, la presencia de comportamientos de hiperreactividad sensorial puede limitar la participación en actividades de sueño en la infancia.

Sin embargo, el valor $r$ obtenido en dichas correlaciones, aun mostrando una correlación positiva $(0<r<1)$, nos indica que la correlación es escasa entre altas puntuaciones en el cuestionario SDSC y el cuadrante de Evitación y Sensibilidad del PS-2 Breve, así como en la sección Sensorial. La correlación es moderada entre las puntuaciones en el SDSC y el cuadrante Búsqueda del PS-2 Breve. Esto supone que aunque la hipótesis planteada al inicio de este estudio queda altamente refutada $(p<0,01)$, es probable que las herramientas utilizadas para detectar problemas de hiperreactividad sensorial, así como alteraciones del sueño en la infancia posean limitaciones que no se han tenido en cuenta. Debemos tener presente que tanto el PS-2 Breve como la SDSC son cuestionarios basados en la percepción de los padres y/o madres, y que en este estudio no se han usado otras técnicas de evaluación propia y directa del niño por parte de los investigadores, lo que puede suponer una limitación a tener en cuenta para el desarrollo de estudios posteriores, que además podrían incluir algún método de recogida de información cualitativa para conocer en su totalidad las percepciones de los padres y/o madres. 
Otra de las limitaciones está relacionada con la muestra ya que, aun siendo amplia, esta es muy heterogénea, tanto por el rango de edad estudiado como por los diversos tipos de diagnósticos médicos incluidos.

No obstante, se considera que desde la Terapia Ocupacional no sólo es importante detectar alteraciones del sueño, sino que se deben crear y usar herramientas para evaluar y detectar problemas en la participación en actividades de sueño.

Se valora como acertado el uso de la SDSC en este estudio, ya que, a diferencia de la mayoría de los cuestionarios similares existentes, abarca un período amplio de tiempo, 6 meses de presencia de los comportamientos señalados, lo que nos puede ayudar a diferenciar problemas transitorios de otras alteraciones del sueño crónicas o persistentes (Bruni, et al., 1996)we developed a 27 item Likert-type rating scale (Sleep Disturbance Scale for Children: SDSC.

Este estudio sugiere la importancia de la falta del uso de otras herramientas de evaluación como el PS-2 Breve, que en conjunto con aquellas que detectan alteraciones en el sueño, puedan formar un diagnóstico más completo de las alteraciones del sueño en la infancia. Esto justifica la importancia de una intervención interdisciplinar, con presencia del terapeuta ocupacional, en los equipos especializados en medicina del sueño, ya que se conseguiría una evaluación más completa que contemple factores que pueden estar influyendo en la participación del niño en actividades de sueño.

Estos primeros resultados respaldan la relación existente entre problemas de hiperreactividad sensorial y alteraciones del sueño en la infancia, propiciando las bases para un futuro estudio transversal en el que se pudieran analizar las relaciones entre la aparición de alteraciones del sueño y de comportamientos de hiperreactividad sensorial en edades tempranas y el impacto del tratamiento de Terapia Ocupacional en dichas alteraciones.

Conociendo estos datos nos planteamos si se consolidan los aprendizajes relacionados a la regulación emocional que los niños realizan durante las intervenciones de Terapia Ocupacional y si es frecuente o no que en la práctica diaria de los terapeutas ocupacionales se estén realizando evaluaciones de la participación en actividades de sueño para conocer si los problemas de regulación emocional están relacionados con dicha ocupación, por lo que sería interesante conocer qué herramientas usan los terapeutas ocupacionales en la práctica clínica para evaluar estos aspectos.

Actualmente en Terapia Ocupacional se usan unos modelos de participación en las actividades de descanso y sueño muy ligados a la productividad del niño durante el día, (AOTA, 2017) obviando aspectos como la relación entre la calidad del sueño, el bienestar y la calidad de vida tanto del niño como de la familia en los momentos del desempeño de las actividades de descanso y sueño.

Con este estudio queremos contribuir al desarrollo de nuevos modelos y marcos para la práctica clínica que contemplen la participación activa e independiente del niño tanto despierto como cuando duerme.

\section{AgRADECIMIENTOS}

Al equipo de Terapeutas Ocupacionales del S.T.O.I. Jerez y a las más de 92 familias que han formado parte de este estudio, sin ellas no hubiera sido posible la obtención de estos resultados. A los terapeutas ocupacionales chilenos por dar la oportunidad de recopilar y publicar los trabajos que realizamos en nuestra práctica diaria.

\section{RefERENCIAS}

Abril, B., Méndez, M., Sans, O., y Valdizán, J.R. (2001). El sueño en el autismo infantil. Revista Neurología, 32(7), 641-644.

Allik, H., Larsson, J.O., y Smedje, H. (2006). Insomnia in school-age children with Asperger syndrome or high-functioning autism. BMC Psychiatry, 6 (18). doi: https://doi.org/10.1186/1471-244X-6-18

American Occupational Therapy Association - AOTA. (2017) Occupational Therapy Practice Framework: domain and process (3rd Edition). The American journal of occupational therapy: official publication of the American Occupational Therapy Association, 68, (1), 1-48. doi: https://doi.org/10.5014/ajot.2014.682006

Brown, D.W. (1996). Autism, Asperger's Syndrome and the CrickMitchison Theory of the Biological Function of REM Sleep. Medical Hypotheses, 47 (5), 399-403. doi: https://doi.org/10.1016/ s0306-9877(96)90220-0

Bruni, O., Romeo, D.M., Brogna, C., Ferri, R., Galluccio, C., De Clemente, V., Di Jorio, M., Quintiliani, M., Ricci, D., y Mercuri, E. (2013). Application of the Sleep Disturbance Scale for Children (SDSC) in preschool age. European Journal of Paediatric Neurology. 17(4), 374-382. doi: https://doi.org/10.1016/j.ejpn.2012.12.009 
Bruni, O., Ottaviano, S., Guidetti, V., Romoli, M., Innocenzi, M., Cortesi, F., y Giannotti, F. (1996). The Sleep Disturbance Scale for Children (SDSC). Construction and validation of aninstrument to evaluate sleepdisturbances in childhood and adolescence. Journal of Sleep Research, 5 (4), 251-261. doi: https://doi.org/10.1111/j.13652869.1996.00251.x

Cousins, J.N., Rijn, E., Ong, J.L., Wong, K.F., y Chee, M.W.L. (2019). Does splitting sleep improve long-term memory in chronically sleep deprived adolescents? Npj Science of Learning, 4 (1). doi: https://doi.org/10.1038/s41539-019-0047-7

Cramer, A.O.J., Waldorp, L.J., Van der Maas, H.L.J., y Borsboom, D. (2010). Comorbidity: A network perspective. Behavioral and Brain Sciences, 33 (2-3), 137-150. doi: https://doi.org/10.1017/ s0140525x09991467

Cruz, IJ. (2018). Alteraciones del sueño infantil. En: AEPap (ed.). Curso de Actualización Pediatría 2018 (pp. 317-329). Madrid, España: Lúa Ediciones.

Dunn, W. (2016). Perfil Sensorial para niños-2. Madrid: Pearson Psycho. Ho, E., y Siu, A. (2018). Occupational Therapy Practice in Sleep Management: A Review of Conceptual Models and Research Evidence. Occupational therapy international. doi: https://doi. org/10.1155/2018/8637498

Foitzik, K., y Brown, T. (2018). Relationship Between Sensory Processing and Sleep in Typically Developing Children. The American journal of occupational therapy: official publication of the American Occupational Therapy Association, 72(1). doi: https://doi.org/10.5014/ ajot.2018.027524

Geiger, A., Achermann, P., y Jenni, O. G. (2010). Association between sleep duration and intelligence scores in healthy children. Developmental psychology, 46(4), 949-954. doi: https://doi. org/10.1037/a0019679

Ghorbanpour, Z., Hosseini, S. A., Akbarfahimi, N., y Rahgozar, M. (2019). Correlation between Sleep Disorders and Function in Children with Spastic Cerebral Palsy. Iranian journal of child neurology, 13(3), 35-44.

Goldman, S. E., Surdyka, K., Cuevas, R., Adkins, K., Wang, L., y Malow, B. A. (2009). Defining the sleep phenotype in children with autism. Developmental neuropsychology, 34(5), 560-573. doi: https://doi.org/10.1080/87565640903133509

Gruber, R., Somerville, G., Enros, P., Paquin, S., Kestler, M., y GilliesPoitras, E. (2014). Sleep efficiency (but not sleep duration) of healthy school-age children is associated with grades in math and languages. Sleep medicine, 15(12), 1517-1525. doi: https://doi. org/10.1016/j.sleep.2014.08.009

Idiazábal-Aletxa, M.A., y Aliagas-Martínez, S. (2009). Sueño en los trastornos del neurodesarrollo. Revista Neurología, 48 (2), 13-16.

Kielhofner, G. (2004). Terapia Ocupacional. Modelo de Ocupación Humana: teoría y aplicación. 3a Ed. Buenos Aires: Medica Panamericana.
Mazurek, M. O., y Petroski, G. F. (2015). Sleep problems in children with autism spectrum disorder: examining the contributions of sensory over-responsivity and anxiety. Sleep medicine, 16(2), 270-279. doi: https://doi.org/10.1016/j.sleep.2014.11.006

Meyer, A. (1922). The philosophy of occupation therapy. American Journal of Physical Medicine \& Rehabilitation, 7(1), 1-10.

Miller, L. J., Anzalone, M. E., Lane, S. J., Cermak, S. A., y Osten, E. T. (2007). Concept evolution in sensory integration: a proposed nosology for diagnosis. The Americanjournal of occupational therapy: official publication of the American Occupational Therapy Association, 67(2), 135-140. doi: https://doi.org/10.5014/ajot.61.2.135

Miller, L. J., McIntosh, D. N., McGrath, J., Shyu, V., Lampe, M., Taylor, A. K., Tassone, F., Neitzel, K., Stackhouse, T., y Hagerman, R. J. (1999). Electrodermal responses to sensory stimuli in individuals with fragile $X$ syndrome: a preliminary report. American journal of medical genetics, 83(4), 268-279.

Ministerio de Sanidad, Política Social e Igualdad. (2013). Guía de Práctica Clínica sobre Trastornos del Sueño en la Infancia y Adolescencia en Atención Primaria. Recuperado de https://www.adolescenciasema. org/wp-content/uploads/2015/07/GPC-sobre-Trastornos-delSue\%C3\%B1o-en-la-Infancia-y-Adolescencia-en-Atenci\%C3\%B3nPrimaria.pdf

O'Brien, L. M., Ivanenko, A., Crabtree, V. M., Holbrook, C. R., Bruner, J. L., Klaus, C. J., y Gozal, D. (2003). The effect of stimulants on sleep characteristics in children with attention deficit/hyperactivity disorder. Sleep medicine, 4(4), 309-316. doi: https://doi. org/10.1016/s1389-9457(03)00071-6

Paavonen, E. J., Räikkönen, K., Pesonen, A. K., Lahti, J., Komsi, N., Heinonen, K., Järvenpää, A. L., Strandberg, T., Kajantie, E., y PorkkaHeiskanen, T. (2010). Sleep quality and cognitive performance in 8-year-old children. Sleep medicine, 11(4), 386-392. doi: https:// doi.org/10.1016/j.sleep.2009.09.009

Reale L., Guarnera M., y Mazzone L. (2014). The effects of sleep disturbance on school performance: A preliminary investigation of children attending elementary grades. School Psychology International, 35 (4), 398-404.

Shani-Adir, A., Rozenman, D., Kessel, A., y Engel-Yeger, B. (2009). The relationship between sensory hypersensitivity and sleep quality of children with atopic dermatitis. Pediatric dermatology, 26(2), 143-149. doi: https://doi.org/10.1111/j.1525-1470.2009.00904.x Shochat, T., Tzischinsky, O., y Engel-Yeger, B. (2009). Sensory hypersensitivity as a contributing factor in the relation between sleep and behavioral disorders in normal schoolchildren. Behavioral sleep medicine, 7(1), 53-62. doi: https://doi.org/10.1080/15402000802577777

Slagle, E. C. (1922). Training aides for mental patients. American Journal of Physical Medicine \& Rehabilitation, 7(1), 11-18.

Valdizán, J. R. (2005). Trastornos generalizados del desarrollo y sueño. Rev Neurol, 47(Supl 1), 135-138.

Vasak, M., Williamson, J., Garden, J., y Zwicker, J. G. (2015). Sensory Processing and Sleep in Typically Developing Infants and Tod- 
dlers. The American journal of occupational therapy: official publication of the American Occupational Therapy Association, 69(4). doi: https://doi.org/10.5014/ajot.2015.015891

Wassing, R., Lakbila-Kamal, O., Ramautar, J. R., Stoffers, D., Schalkwijk, F., y Van Someren, E. (2019). Restless REM Sleep Impedes Overnight Amygdala Adaptation. Current biology: CB, 29(14), 2351-2358. doi: https://doi.org/10.1016/j.cub.2019.06.034 